\title{
Digital IIR Filter Design Using Adaptive Simulated Annealing
}

\author{
S. Chen, ${ }^{*}$ R. Istepanian, ${ }^{\dagger}$ and B. L. Luk ${ }^{\ddagger}$ \\ * Department of Electronics and Computer Science, University of \\ Southampton, Highfield, Southampton SO17 1BJ, United Kingdom; \\ ${ }^{\dagger}$ Department of Electrical and Computer Engineering, Ryerson Polytechnic \\ University, Toronto, Ontario, Canada M5B 2K3; and ${ }^{\text {Center for Intelligent }}$ \\ Design, Automation and Manufacturing, City University of Hong Kong, \\ Kowloon, Hong Kong, People's Republic of China \\ E-mail:sqc@ecs.soton.ac.uk
}

Chen, S., Istepanian, R., and Luk, B. L., Digital IIR Filter Design Using Adaptive Simulated Annealing, Digital Signal Processing 11 (2001) 241251.

Adaptive infinite-impulse-response (IIR) filtering provides a powerful approach for solving a variety of practical problems. Because the error surface of IIR filters is generally multimodal, global optimization techniques are required in order to avoid local minima. We apply a global optimization method, called the adaptive simulated annealing (ASA), to digital IIR filter design. An important advantage of the ASA is the simplicity in software programming. Simulation study involving system identification application shows that the proposed approach is accurate and has a fast convergence rate, and the results obtained demonstrate that the ASA offers a viable tool to digital IIR filter design. $\odot 2001$ Academic Press

Key Words: IIR filter; optimization; adaptive simulated annealing; system identification.

\section{INTRODUCTION}

Adaptive infinite-impulse-response (IIR) filtering has been an active area of research for many years, and many properties of IIR filters are well known [1,2]. A major concern in IIR filtering applications is that the cost function of IIR filters is generally multimodal with respect to the filter coefficients, and the usual gradient-based algorithm can easily be stuck at local minima. In order to achieve a global minimum solution, global optimization techniques are needed. Global optimization methods require extensive computations and are usually batch-type algorithms, as the cost function employed must be evaluated on a block of data. In contrast, gradient- 
based learning can be implemented recursively to update the filter coefficients as each new data sample is acquired. Despite these drawbacks, applying global optimization methods to IIR filter design is attractive, since in many applications a global optimal solution can be much better than local optimal ones.

When considering global optimization methods for digital IIR filter design, the genetic algorithm (GA) [3-5] seems to have attracted considerable attention [6-8]. Simulated annealing (SA) [9-11] by contrast has not received similar interest in this application. SA represents a general global optimization technique with some strikingly positive and negative features. An attractive feature of SA is that it is very easy to program and the algorithm typically has few parameters that require tuning. A serious drawback of SA is that the standard SA algorithms can be very slow, often requiring much more number of cost-function evaluations to converge, compared with a carefully designed and tuned GA.

An improved version of SA, referred to as the adaptive SA (ASA) [12-15], is known to provide significant improvement in convergence speed over standard versions of SA. This ASA is also known as the very fast simulated reannealing. In this study, we apply the ASA to digital IIR filter design. Simulation involving system identification application is used to illustrate the effectiveness of the ASA. A batch-recursive version of the ASA is proposed for adaptive applications. No attempt has been made to explicitly compare the convergence speed of the ASA with that of the GA. However, the examples used are well known and, compared with the results of using GAs for IIR filtering available in the literature, the efficiency of the ASA appears to be on the same order as GA. This suggests that the ASA offers a viable alternative to digital IIR filter design.

\section{THE ASA FOR DIGITAL IIR FILTER DESIGN}

Consider the digital IIR filter with the input-output relationship governed by the difference equation

$$
y(k)+\sum_{i=1}^{M} b_{i} y(k-i)=\sum_{i=0}^{L} a_{i} x(k-i)
$$

where $x(k)$ and $y(k)$ are the filter's input and output, respectively, and $M(\geq L)$ is the filter order. The transfer function of this IIR filter is

$$
H_{M}(z)=\frac{A(z)}{B(z)}=\frac{\sum_{i=0}^{L} a_{i} z^{-i}}{1+\sum_{i=1}^{M} b_{i} z^{-i}} .
$$

The IIR filter design can be formulated as an optimization problem with the mean square error (MSE) as the cost function

$$
J\left(\mathbf{w}_{H}\right)=\mathrm{E}\left[e^{2}(k)\right]=\mathrm{E}\left[(d(k)-y(k))^{2}\right]
$$


where $d(k)$ is the filter's desired response, $e(k)=d(k)-y(k)$ is the filter's error signal, and

$$
\mathbf{w}_{H}=\left[\mathbf{a}^{T} \mathbf{b}^{T}\right]^{T}=\left[\begin{array}{lllllll}
a_{0} & a_{1} & \ldots & a_{L} & b_{1} & \ldots & b_{M}
\end{array}\right]^{T}
$$

denotes the filter coefficient vector. The goal is to minimize the MSE (3) by adjusting $\mathbf{w}_{H}$. In practice, ensemble operation is difficult to realize, and the cost function (3) is usually substituted by the time-averaged cost function:

$$
J_{N}\left(\mathbf{w}_{H}\right)=\frac{1}{N} \sum_{k=1}^{N} e^{2}(k) .
$$

An important consideration during the adaptive process is to maintain the stability of the IIR filter. The IIR filter (1) is in the direct form. An efficient way of maintaining stability is to convert the direct form to the lattice form [16] and to make sure that all the reflection coefficients $\kappa_{i}, 0 \leq i \leq M-1$, have magnitudes less than 1 . We will adopt this approach to guarantee the stability of the IIR filter during adaptation. Thus the actual filter coefficient vector used in optimization is

$$
\mathbf{w}=\left[\begin{array}{llllllll}
a_{0} & a_{1} & \ldots & a_{L} & \kappa_{0} & \ldots & \kappa_{M-1}
\end{array}\right]^{T}=\left[\begin{array}{llll}
w_{1} & \ldots & w_{D}
\end{array}\right]^{T},
$$

where $D=M+L+1$ is the dimension of the filter coefficient vector. For the notational convenience, the cost function will still be denoted as $J$ or $J_{N}$. Converting the reflection coefficients back to the direct-form coefficients $b_{i}$, $1 \leq i \leq M$, is straightforward [16]. For instance, given $M=3$, we have

$$
\left.\begin{array}{l}
b_{3}=\kappa_{2} \\
b_{2}=\kappa_{0}\left(1+\kappa_{1}\right) \kappa_{2}+\kappa_{1} \\
b_{1}=\kappa_{0}\left(1+\kappa_{1}\right)+\kappa_{1} \kappa_{2}
\end{array}\right\} .
$$

Because the cost function for IIR filters is generally multimodal, using a global optimization method, such as ASA, is desired. Theoretic fundamentals and convergence analysis of the ASA algorithm can be found in [12-14] and the references therein. The appendix summarizes the search mechanisms of the ASA method. Although there are many possible realizations of ASA, an implementation of the algorithm is illustrated in Fig. 1, and this algorithm is detailed as follows:

(i) In the initialization, an initial $\mathbf{w}$ is randomly generated, the initial temperature of the acceptance probability function, $T_{c}(0)$, is set to the initial value of the cost function $J(\mathbf{w})$, and the initial temperatures of the parameter generating probability functions, $T_{i}(0), 1 \leq i \leq D$, are set to 1.0. A user-defined control parameter $c$ in annealing process is given, and the annealing times, $k_{i}$ for $1 \leq i \leq D$ and $k_{c}$, are all set to 0 . 


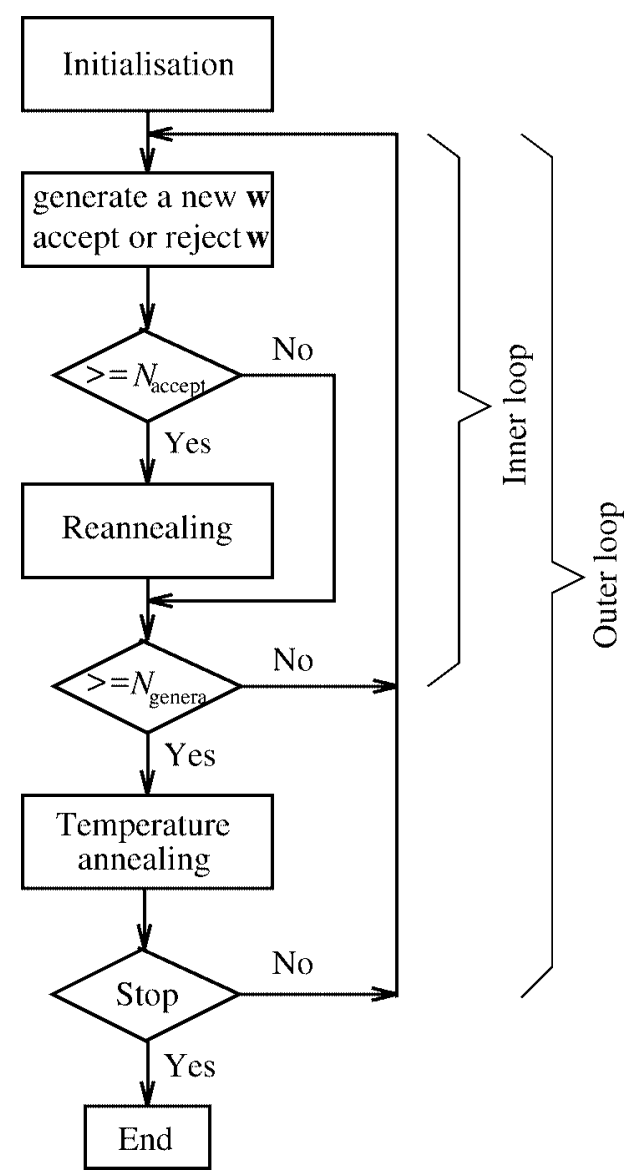

FIG. 1. Flow chart of the adaptive simulated annealing.

(ii) The algorithm generates a new point in the parameter space with

$$
w_{i}^{\text {new }}=w_{i}^{\text {old }}+g_{i}\left(U_{i}-V_{i}\right) \quad \text { and } \quad w_{i}^{\text {new }} \in\left[U_{i}, V_{i}\right], \quad \text { for } 1 \leq i \leq D .
$$

Here $U_{i}$ and $V_{i}$ are the lower and upper bounds for $w_{i}, g_{i}$ is calculated as

$$
g_{i}=\operatorname{sgn}\left(u_{i}-\frac{1}{2}\right) T_{i}\left(k_{i}\right)\left(\left(1+\frac{1}{T_{i}\left(k_{i}\right)}\right)^{\left|2 u_{i}-1\right|}-1\right),
$$

and $u_{i}$ a uniformly distributed random variable in $[0,1]$. The value of the cost function $J\left(\mathbf{w}^{\text {new }}\right)$ is then evaluated and the acceptance probability function of $\mathbf{w}^{\text {new }}$ is given by

$$
P_{\text {accept }}=\frac{1}{1+\exp \left(\left(J\left(\mathbf{w}^{\text {new }}\right)-J\left(\mathbf{w}^{\text {old }}\right)\right) / T_{c}\left(k_{c}\right)\right)} .
$$

A uniform random variable $P_{\text {unif }}$ is generated in $[0,1]$. If $P_{\text {unif }} \leq P_{\text {accept }}, \mathbf{w}^{\text {new }}$ is accepted; otherwise it is rejected. 
(iii) After every $N_{\text {accept }}$ acceptance points, reannealing takes place by first calculating the sensitivities

$$
s_{i}=\left|\frac{J\left(\mathbf{w}^{\text {best }}+\mathbf{1}_{i} \delta\right)-J\left(\mathbf{w}^{\text {best }}\right)}{\delta}\right|, \quad 1 \leq i \leq D,
$$

where $\mathbf{w}^{\text {best }}$ is the best point found so far, $\delta$ is a small step size, the $D$ dimensional vector $\mathbf{1}_{i}$ has unit $i$ th element and the rest of elements of $\mathbf{1}_{i}$ are all zeros. Let $s_{\max }=\max \left\{s_{i}, 1 \leq i \leq D\right\}$. Each $T_{i}$ is scaled by a factor $s_{\max } / s_{i}$ and the annealing time $k_{i}$ is reset

$$
T_{i}\left(k_{i}\right)=\frac{s_{\max }}{s_{i}} T_{i}\left(k_{i}\right), \quad k_{i}=\left(-\frac{1}{c} \log \left(\frac{T_{i}\left(k_{i}\right)}{T_{i}(0)}\right)\right)^{D} .
$$

Similarly, $T_{c}(0)$ is reset to the value of the last accepted cost function, $T_{c}\left(k_{c}\right)$ is reset to $J\left(\mathbf{w}^{\text {best }}\right)$, and the annealing time $k_{c}$ is rescaled accordingly:

$$
k_{c}=\left(-\frac{1}{c} \log \left(\frac{T_{c}\left(k_{c}\right)}{T_{c}(0)}\right)\right)^{D} .
$$

(iv) After every $N_{\text {genera }}$ generated points, annealing takes place with

$$
\left.\begin{array}{rl}
k_{i} & =k_{i}+1 \\
T_{i}\left(k_{i}\right) & =T_{i}(0) \exp \left(-c k_{i}^{1 / D}\right)
\end{array}\right\} 1 \leq i \leq D
$$

and

$$
\left.\begin{array}{rl}
k_{c} & =k_{c}+1 \\
T_{c}\left(k_{c}\right) & =T_{c}(0) \exp \left(-c k_{c}^{1 / D}\right)
\end{array}\right\} .
$$

Otherwise, go to step (ii).

(v) The algorithm is terminated if the parameters have remained unchanged for a few successive reannealings or a preset maximum number of costfunction evaluations have been reached; otherwise, go to step (ii).

As in a standard SA, the ASA contains two loops. The inner loop ensures that the parameter space is searched sufficiently at a given temperature, which is necessary to guarantee that the algorithm finds a global optimum. The differences with standard SAs are that the ASA uses a much faster annealing schedule and employs a reannealing scheme to adapt itself. The ASA is easy to program, and the user only needs to assign a control parameter $c$ and set two values $N_{\text {accept }}$ and $N_{\text {genera }}$.

The above ASA is basically a batch algorithm. For adaptive applications, it is desired to have some tracking capability. This can be achieved by employing a moving window scheme, as illustrated in Fig. 2. The cost function is evaluated using a block of $N$ samples but the data block is shifted by $\Delta N$ samples after every $\Delta N$ cost-function evaluations. We will refer to this version of the algorithm as the batch-recursive algorithm. 


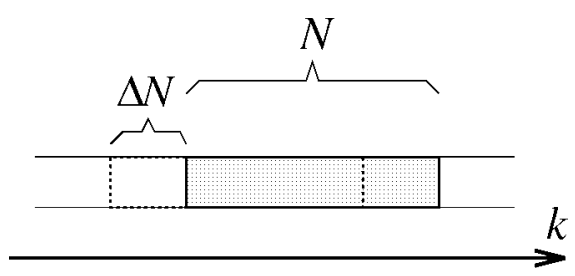

FIG. 2. Illustration of the batch-recursive method with a moving window. The data block contains $N$ samples and, after every $\Delta N$ cost-function evaluations, the data block is shifted by $\Delta N$ samples.

\section{SYSTEM IDENTIFICATION APPLICATION}

System identification application, depicted in Fig. 3, is used in the experiment. In this configuration, the unknown plant has a transfer function $H_{S}(z)$, and the ASA is employed to adjust the IIR filter that is used to model the system. When the filter order $M$ is smaller than the system order, local minima problems can be encountered [2], and this is used to simulate a multimodal environment. We also apply the standard, batch gradient algorithm in the simulation to highlight the problems caused by local minima. The batch gradient algorithm is defined by

$$
\mathbf{w}^{(l+1)}=\mathbf{w}^{(l)}-\alpha \frac{\partial J\left(\mathbf{w}^{(l)}\right)}{\partial \mathbf{w}} .
$$

A variable gain $\alpha$ is adopted for fast convergence. For the cost function (5), 2000 samples are used in averaging for the batch ASA and gradient algorithms. The signal-to-noise (SNR) ratio of the system is defined as

$$
\mathrm{SNR}=\frac{\sigma_{s}^{2}}{\sigma_{n}^{2}} .
$$

Here $\sigma_{n}^{2}$ is the noise variance, and the system signal variance $\sigma_{s}^{2}$ is given by

$$
\sigma_{s}^{2}=\frac{\sigma_{x}^{2}}{j} \oint H_{S}(z) H_{S}\left(z^{-1}\right) \frac{d z}{z},
$$

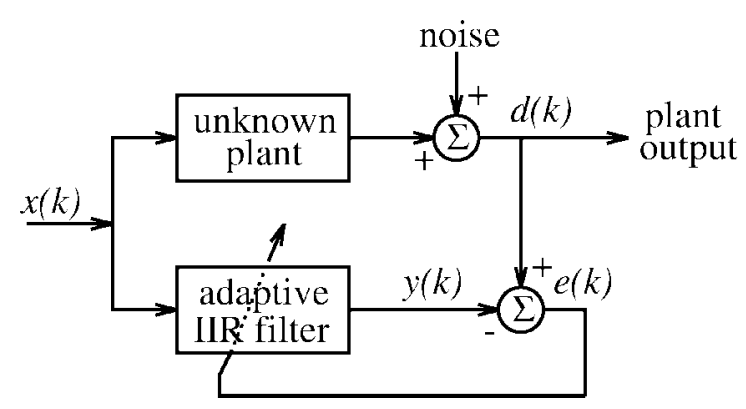

FIG. 3. Schematic of adaptive IIR filter for system identification. 


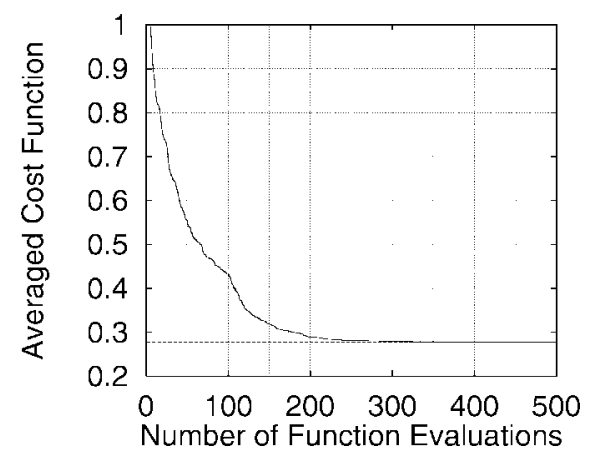

FIG. 4. Normalized cost function versus number of cost-function evaluations averaged over 100 random runs of the batch ASA for Example 1. Each run had a randomly chosen initial point. The dashed line indicates the global minimum.

where $j=\sqrt{-1}, \sigma_{x}^{2}$ is the input variance, and a numerical evaluation of the filter power $\oint H_{S}(z) H_{S}\left(z^{-1}\right) d z / z$ can be found in [17]. The search ranges for the filter coefficients are $\left|a_{i}\right| \leq 1.2$ and $\left|\kappa_{i}\right| \leq 0.99$.

ExAmple 1. This example is taken from [2]. The system and filter transfer functions are respectively

$$
H_{S}(z)=\frac{0.05-0.4 z^{-1}}{1-1.1314 z^{-1}+0.25 z^{-2}}, \quad H_{M}(z)=\frac{a_{0}}{1+b_{1} z^{-1}} .
$$

The analytical cost function $J$ in this case is known when the input is a white sequence and $\sigma_{n}^{2}=0$. The cost function has a global minimum at $\mathbf{w}^{\text {global }}=$ $[-0.311-0.906]^{T}$ with the value of the normalized cost function $J\left(\mathbf{w}^{\text {global }}\right) / \sigma_{s}^{2}=$ 0.2772 and a local minimum at $\mathbf{w}^{\text {local }}=\left[\begin{array}{lll}0.114 & 0.519\end{array}\right]^{T}$. The batch gradient algorithm cannot find $\mathbf{w}^{\text {global }}$ when the initial $\mathbf{w}$ is near $\mathbf{w}^{\text {local }}$. Figure 4 depicts the evolution of the normalized cost function averaged over 100 different random runs of the batch ASA. Each run had a randomly chosen initial $\mathbf{w}$ and a random algorithm setting. Figure 5 shows the trajectories of the filter parameter vector averaged over 100 different runs of the batch ASA, started from four fixed initial positions. It can be seen that the batch ASA consistently found the global optimal solution and the algorithm converged after 300 function calls. The ability of the ASA to escape from a local minimum is clearly demonstrated by this example.

EXAMPLE 2. This is a third-order system with the transfer function given by

$$
H_{S}(z)=\frac{-0.3+0.4 z^{-1}-0.5 z^{-2}}{1-1.2 z^{-1}+0.5 z^{-2}-0.1 z^{-3}} .
$$

In the simulation, the system input $x(k)$ was a uniformly distributed white sequence, taking values from $(-0.5,0.5)$, and the $\mathrm{SNR}=30 \mathrm{~dB}$. When the filter order was equal to the system order, both the batch ASA and gradient algorithms converged to the global optimum with the MSE $J_{N}$ reaching the 


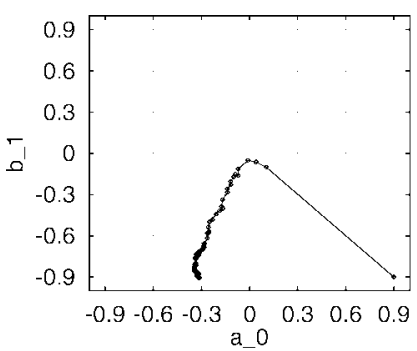

(a)

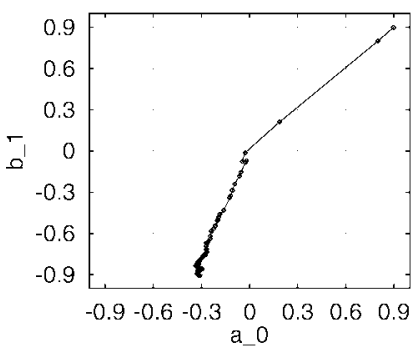

(c)

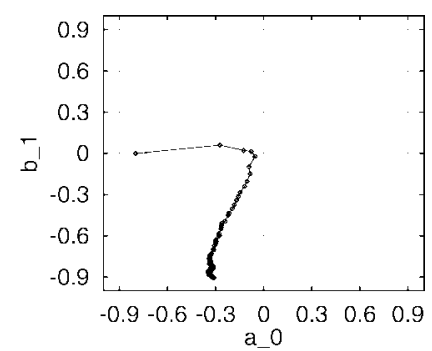

(b)

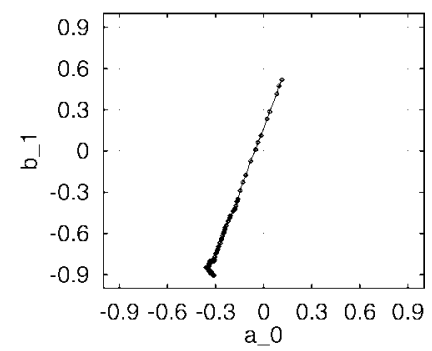

(d)

FIG. 5. Trajectories of the filter parameter vector averaged over 100 different runs of the batch ASA, started from the fixed initial positions: (a) $\left[\begin{array}{lll}0.9 & -0.9\end{array}\right]^{T}$, (b) $\left[\begin{array}{ll}-0.8 & 0.0\end{array}\right]^{T}$, (c) $\left[\begin{array}{ll}0.9 & 0.9\end{array}\right]^{T}$, and (d) $[0.1140 .519]^{T}$ for Example 1 .

noise floor of 0.0002 . The gradient algorithm in this case was much more efficient than the ASA. However, when a reduced-order filter with $M=2$ and $L=1$ was used, the MSE became multimodal and the gradient algorithm converged to the two final states, depending on the initial conditions. Figure 6 shows the behaviors of the batch gradient algorithm when started from the two quite closed initial positions. The batch ASA consistently reached the global

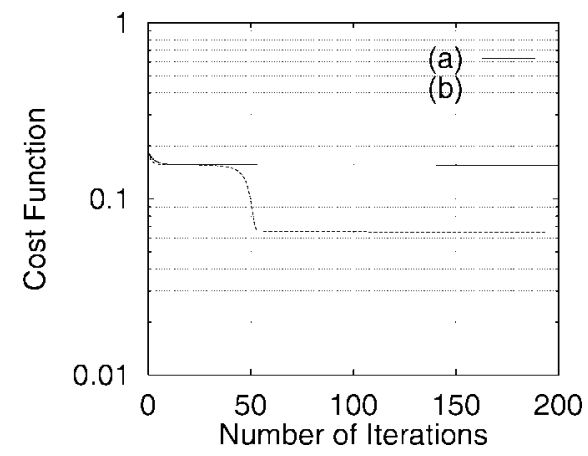

FIG. 6. Convergence behaviors of the batch gradient algorithm, started from the two initial conditions: (a) $\left[\begin{array}{lllll}0.0 & 0.0 & 0.3 & 0.1\end{array}\right]^{T}$ and (b) $\left[\begin{array}{llll}0.0 & 0.0 & 0.3 & 0.0\end{array}\right]^{T}$ for Example 2. 


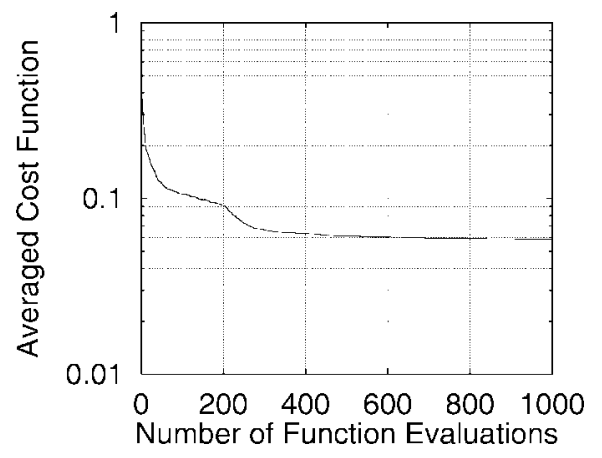

FIG. 7. Cost function versus number of cost-function evaluations averaged over 100 random runs of the batch ASA for Example 2. Each run had a randomly chosen initial point.

optimal solution, as shown in Fig. 7. The batch-recursive ASA algorithm was also tested for this example. We used a moving window of $N=100$ and $\Delta N=1$. Figure 8 plots the evolution of the cost function averaged over 100 different random runs of the batch-recursive ASA. Again each run had a randomly chosen initial $\mathbf{w}$ and a random algorithm setting.

\section{CONCLUSIONS}

Although the digital IIR filter design is a well-researched area, major difficulties still exist in practice. An efficient global optimization method known as the ASA has been applied to overcome the problems associated with local minima. Simulation study involving system identification application has demonstrated that the ASA is robust and has a fast convergence speed. Compared with the results of using GAs for adaptive IIR filtering available in the literature, the efficiency of the ASA appears at least to be on the same

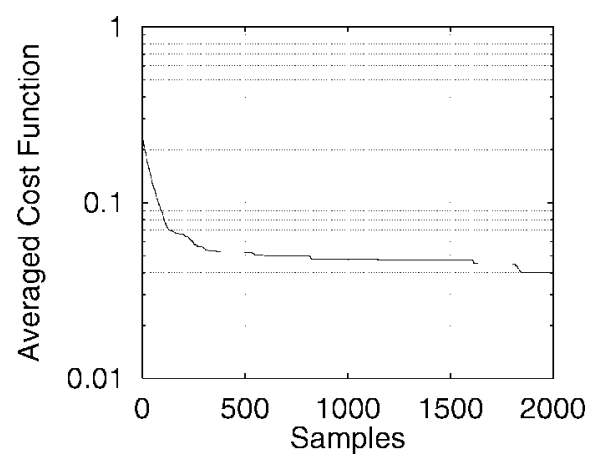

FIG. 8. Cost function versus number of cost-function evaluations averaged over 100 random runs of the batch-recursive ASA for Example 2. A moving window of $N=100$ and $\Delta N=1$ was used. Each run had a randomly chosen initial point. 
order as GA. This study has confirmed that the ASA offers an alternative design approach for IIR filtering.

\section{APPENDIX: ASA SEARCH GUIDING MECHANISMS}

The ASA is a global optimization scheme for solving for the following general optimization problem:

$$
\min _{\mathbf{w} \in \mathcal{W}} J(\mathbf{w})
$$

It evolves a single point $\mathbf{w}$ in the parameter or state space $\mathcal{W}$. The seemingly random search is guided by certain underlying probability distributions. Specifically, the general SA algorithm is described by three functions.

\section{Generating Probability Density Function}

$$
G\left(w_{i}^{\text {old }}, w_{i}^{\text {new }}, T_{i} ; 1 \leq i \leq D\right) .
$$

This determines how a new state $\mathbf{w}^{\text {new }}$ is created and from what neighborhood and probability distributions it is generated given the current state $\mathbf{w}^{\text {old }}$. The generating "temperatures" $T_{i}$ describe the widths or scales of the generating distribution along each dimension $w_{i}$ of the state space.

Often a cost function has different sensitivities along different dimensions of the state space. Ideally, the generating distribution used to search a steeper and more sensitive dimension should have a narrower width than that of the distribution used in searching a dimension less sensitive to change. The ASA adopts a so-called reannealing scheme to periodically rescale $T_{i}$, so that they optimally adapt to the current status of the cost function. This is an important mechanism, which not only speeds up the search process but also makes the optimization process robust to different problems.

\section{Acceptance Function}

$$
P_{\text {accept }}\left(J\left(\mathbf{w}^{\text {old }}\right), J\left(\mathbf{w}^{\text {new }}\right), T_{c}\right) .
$$

This gives the probability of $\mathbf{w}^{\text {new }}$ being accepted. The acceptance temperature $T_{c}$ determines the frequency of accepting new states of poorer quality.

Probability of acceptance is very high at very high temperature $T_{c}$, and it becomes smaller as $T_{c}$ is reduced. At every acceptance temperature, there is a finite probability of accepting the new state. This produces an occasionally uphill move, enables the algorithm to escape from local minima, and allows a more effective search of the state space to find a global minimum. The ASA also periodically adapts $T_{c}$ to best suit the status of the cost function. This helps to improve convergence speed and robustness. 


\section{Reduce Temperatures or Annealing Schedule}

$$
\left.\begin{array}{l}
T_{c}\left(k_{c}\right) \rightarrow T_{c}\left(k_{c}+1\right) \\
T_{i}\left(k_{i}\right) \rightarrow T_{i}\left(k_{i}+1\right), 1 \leq i \leq D
\end{array}\right\},
$$

where $k_{c}$ and $k_{i}$ are some annealing time indexes. The reduction of temperatures should be sufficiently gradual in order to ensure that the algorithm finds a global minimum.

This mechanism is based on the observations of the physical annealing process. When the metal is cooled from a high temperature, if the cooling is sufficiently slow, the atoms line themselves up and form a crystal, which is the state of minimum energy in the system. The slow convergence of many SA algorithms is rooted at this slow annealing process. The ASA, however, can employ a very fast annealing schedule, as it has self adaptation ability to rescale temperatures.

\section{REFERENCES}

1. Widrow, B. and Stearns, S. D., Adaptive Signal Processing. Prentice Hall International, Englewood Cliffs, NJ, 1985.

2. Shynk, J. J., Adaptive IIR filtering. IEEE ASSP Mag. April (1989), 4-21.

3. Holland, J. H., Adaptation in Natural and Artificial Systems. Univ. of Michigan Press, Ann Arbor, 1975.

4. Goldberg, D. E., Genetic Algorithms in Search, Optimization, and Machine Learning. AddisonWesley, Reading, MA, 1989.

5. Davis, L. (Ed.), Handbook of Genetic Algorithms. Reinhold, New York, 1991.

6. Nambiar, R., Tang, C. K. K., and Mars, P., Genetic and learning automata algorithms for adaptive digital filters. In Proc. ICASSP, 1992, Vol. IV, pp. 41-44.

7. Wilson, P. B. and Macleod, M. D., Low implementation cost IIR digital filter design using genetic algorithms. In Workshop on Natural Algorithms in Signal Processing, Chelmsford, Essex, 1993, pp. $4 / 1-4 / 8$.

8. Ng, S. C., Leung, S. H., Chung, C. Y., Luk, A., and Lau, W. H., The genetic search approach: A new learning algorithm for adaptive IIR filtering. IEEE Signal Process. Mag. November (1996), 3846.

9. Kirkpatrick, S., Gelatt, Jr., C. D., and Vecchi, M. P., Optimization by simulated annealing. Science 220 (1983), 671-680.

10. Corana, A., Marchesi, M., Martini, C., and Ridella, S., Minimizing multimodal functions of continuous variables with the simulated annealing algorithm. ACM Trans. Math. Software 13 (1987), 262-280.

11. van Laarhoven, P. J. M. and Aarts, E. H. L., Simulated Annealing: Theory and Applications. Reidel, Dordrecht, 1987.

12. Ingber, L. and Rosen, B., Genetic algorithms and very fast simulated reannealing: A comparison. Math. Comput. Model. 16 (1992), 87-100.

13. Ingber, L., Simulated annealing: Practice versus theory. Math. Comput. Model. 18 (1993), $29-57$.

14. Ingber, L., Adaptive simulated annealing (ASA): Lessons learned. J. Control Cybernet. 25 (1996), 33-54.

15. Chen, S., Luk, B. L., and Liu, Y., Application of adaptive simulated annealing to blind channel identification with HOC fitting. Electron. Lett. 34 (1998), 234-235.

16. Gray, Jr., A. H. and Markel, J. D., Digital lattice and ladder filter synthesis. IEEE Trans. Audio Electroacoust. AU-21 (1973), 491-500.

17. Åström, K. J., Introduction to Stochastic Control Theory. Academic Press, New York, 1970. 